(C) 2018. This manuscript version is made available under the CC-BY-NC-ND 4.0 license http:/creativecommons.org/licenses/by-nc-nd/4.0/

\title{
Ultrafast Laplace NMR with hyperpolarized xenon gas
}

Otto Mankinen ${ }^{\mathrm{a}}$, Julia Hollenbach ${ }^{\mathrm{b}}$, Susanna Ahola ${ }^{\mathrm{a}}$, Jörg Matysik ${ }^{\mathrm{b}}$, Ville-Veikko Telkki $^{\mathrm{a}^{*}}$

${ }^{a}$ NMR Research Unit, University of Oulu, P.O. Box 3000, FIN-90014, Finland

${ }^{b}$ Institut für Analytische Chemie, Universität Leipzig, Linnéstr. 3, D-04103 Leipzig, Germany

* Corresponding Author.

E-mail address: ville-veikko.telkki@oulu.fi (V.-V. Telkki) 


\begin{abstract}
Laplace NMR, consisting of diffusion and relaxation experiments, provides detailed information about dynamics of fluids in porous materials. Recently, we showed that two-dimensional Laplace NMR experiments can be carried out with a single scan based on spatial encoding. The method shortens the experiment time by one to three orders of magnitude, and therefore it is called ultrafast Laplace NMR. Furthermore, the singlescan approach facilitates significantly the use of nuclear spin hyperpolarization for boosting the sensitivity of the experiment, because a laborious hyperpolarization procedure does not need to be repeated. Here, we push the limits of the ultrafast Laplace NMR method by applying it, for the first time, in the investigation of a gas phase substance, namely hyperpolarized xenon gas. We show that, regardless of the fast diffusion of gas, layer-like spatial encoding is feasible, and an ultrafast diffusion $-T_{2}$ relaxation correlation experiment reveals significantly different signals of free gas and gas adsorbed in a mesoporous controlled pore glass (CPG). The observed diffusion coefficients are many orders of magnitude larger than those detected earlier from liquid phase substances, emphasizing the extended application range of the method. The challenges in the methodology, caused by the fast diffusion, are also discussed.
\end{abstract}




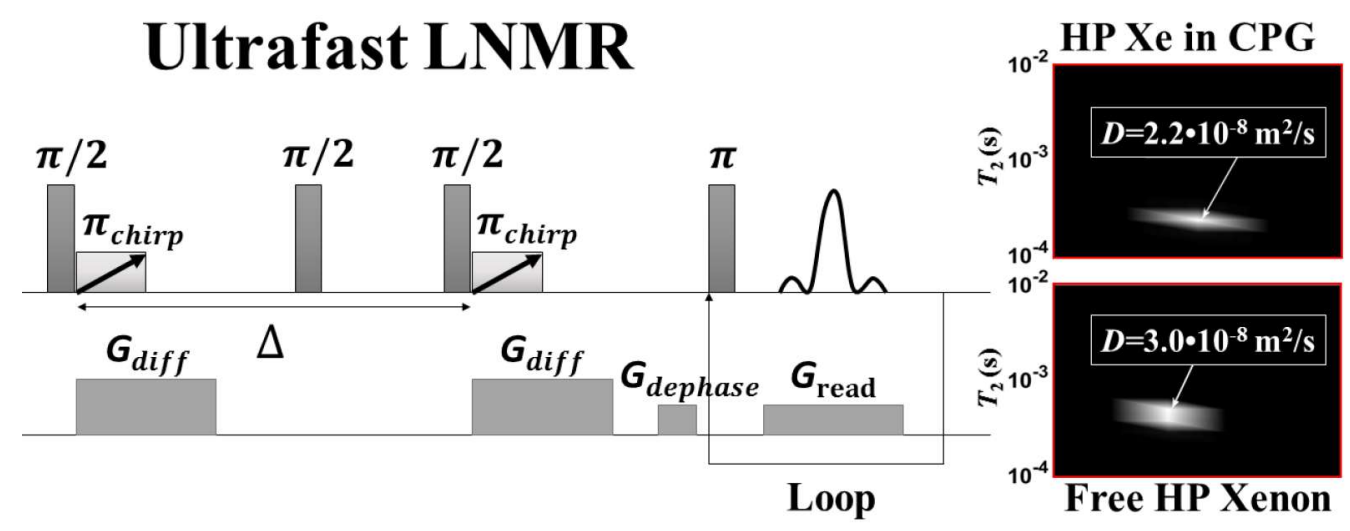

Highlights:

- The first gas phase ultrafast Laplace NMR

- Significant sensitivity enhancement with hyperpolarized xenon

- Differences in the dynamics of free and adsorbed gas

Keywords:

Ultrafast Laplace NMR

Relaxation

Diffusion

Mesoporous materials

Hyperpolarized xenon 


\section{Introduction}

Nuclear magnetic resonance (NMR) relaxation and diffusion experiments provide unique information about the rotational and translational motion of molecules as well as local chemical environment of nuclei $[1,2]$. Because radio frequency waves used in NMR penetrate through optically opaque materials, NMR is an excellent non-invasive method for the investigation of the structure of materials as well as of dynamics of fluids confined to porous materials [3-6].

Relaxation and diffusion experiments result in exponentially decaying signal intensities and the distribution of relaxation times or diffusion coefficients is extracted from the data by a mathematical process resembling an inverse Laplace transform [2]. Therefore, the experiments are classified under the concept of Laplace NMR (LNMR).

A multidimensional approach significantly improves both the resolution and information content of LNMR, enabling the correlation of diffusion and relaxation parameters as well as investigation of chemical exchange phenomena even in the case when the exchanging sites are not resolved in conventional spectra $[2,5,7]$. The approach has become broadly available after the development of a sufficiently reliable and robust multidimensional Laplace inversion algorithm [8-10]. The multidimensional approach increases the experiment time significantly, because the experiment has to be repeated from tens to hundreds of times in order to collect data corresponding to each indirect dimension [2]. The approach also complicates the use of nuclear spin hyperpolarization techniques [11-14] for improving the sensitivity of experiments, because the polarization has to be reproduced again before each repetition, being laborious and time-consuming. 
Recently, we introduced the ultrafast multidimensional LNMR approach as an answer to these drawbacks [15-17]. The method is based on similar layer-like spatial encoding of multidimensional data that has earlier been exploited in ultrafast multidimensional NMR spectroscopy [18-20] (also with hyperpolarization [21]), in 1D LNMR experiments [22-24], as well as for correlating $T_{1}$ relaxation or diffusion parameters with spectroscopic data [25-27]. With this approach, we have shown that single-scan multidimensional LNMR experiments become feasible [15,16]. Although the spatial encoding lowers the sensitivity of the experiment, the sensitivity per unit time is slightly increased $[15,17]$, and we were able to improve the overall sensitivity by several orders of magnitude with the use of hyperpolarized substances [16]. Ultrafast $T_{1}-T_{2}$ and $D-T_{2}$ relaxation correlation experiments revealed, e.g., different physical environments of liquid molecules in porous materials [15-17], and we showed that the experiments are feasible even with a low field, single-sided NMR instrument [17].

To the best of our knowledge, up to now only liquid-state ultrafast NMR spectroscopy and LNMR measurements has been reported in the literature, however, no gas phase experiments have been provided. There is an obvious reason for that: fast diffusion of gases may mix the spatial, layer-like data encoding. The problem is emphasized in ultrafast NMR spectroscopy based on gradient-echo-type reading of the data [20], being sensitive to magnetic field inhomogeneities. Ultrafast LNMR, however, is based on the use of spin-echoes [2] that refocuses the effect of magnetic field inhomogeneity, and successful gas phase experiments will be more plausible with the ultrafast LNMR than with ultrafast NMR spectroscopy.

This work aims at extending the application range of ultrafast LNMR to gas phase substances, regardless of their large diffusion coefficient and low spin density. The 
studies concentrate on xenon gas, because its ${ }^{129} \mathrm{Xe}$ isotope has a spin-1/2 nucleus and relatively high natural abundance $(26 \%)$. Furthermore, nuclear spin polarization of ${ }^{129} \mathrm{Xe}$ can be enhanced by many orders of magnitude by spin-exchange optical pumping (SEOP) technique $[13,28] .{ }^{129} \mathrm{Xe}$ has also turned out to be an excellent probe for the properties of porous materials [29-33]. Here we describe the first gas phase ultrafast LNMR (diffusion$T_{2}$ correlation) experiments for hyperpolarized free xenon gas as well as xenon gas in a mesoporous material.

\section{Experimental}

NMR experiments were carried out on Bruker DRX 400 spectrometer equipped with a 5-mm broad band probe operating at $110.68 \mathrm{MHz}$ for ${ }^{129} \mathrm{Xe}$. Experiments were conducted at room temperature. The hyperpolarized (HP) ${ }^{129} \mathrm{Xe}$ gas was produced with a home-built continuous-flow Xe-polarizer, using a gas mixture consisting of $2.4 \%$ $\mathrm{Xe}$ (with natural isotopic portions), $9.6 \% \mathrm{~N}_{2}$ and $88 \% \mathrm{He}$. The optical pumping cell made of Pyrex contained about $300 \mathrm{mg} \mathrm{Rb}$ (purity: $>99 \%$, AlfaAesar) and was operated at $145^{\circ}$ $\mathrm{C}$ and 3 bar for the pumping process. For the experiments, the gas mixture containing the $\mathrm{HP}^{129} \mathrm{Xe}$ was directly inserted into standard $5 \mathrm{~mm}$ o.d. NMR tubes using a homedesigned gas-insertion cap. The pressure of the gas mixture in the sample tube during the experiments was around 1 bar. The gas flow through the sample was regulated by a valve system controlled via a solenoid valve, allowing to stop the flow during the measurement. An illustration of the experimental setup is shown in Fig. 1a.

Ultrafast $D-T_{2}$ correlation measurements of free hyperpolarized xenon gas and hyperpolarized xenon inside a mesoporous controlled pore glass $(\mathrm{CPG}$, provided by 
Enke et al., University of Leipzig), with the average pore diameter of $13 \mathrm{~nm}$ and particle size of $200-250 \mu \mathrm{m}$, were carried out. Prior to the experiments, the sample was dried at $160{ }^{\circ} \mathrm{C}$ for 48 hours. The pulse sequence is shown in Fig. 1b. As explained in detail in ref. [16], after the two diffusion gradients the diffusion decay curve is encoded into the transverse magnetization profile along the $z$ axis, and then the profile is read in the CPMG loop by means of read gradients, similar to magnetic resonance imaging (MRI) [3]. Between the successive echoes, the profile is decaying due to $T_{2}$ relaxation.

In the free gas experiment, the following parameters were used: the duration and the bandwidth of the frequency-swept chirp pulse were $1 \mathrm{~ms}$ and $8266 \mathrm{~Hz}$. The diffusion delay $(\Delta)$ was $4.5 \mathrm{~ms}$ and the strength of the diffusion gradient ( $G_{\text {diff }}$ ) was 0.033 $\mathrm{T} / \mathrm{m}$. The echo time in the CPMG loop was $6.5 \mathrm{~ms}$, the strength of the read gradient $\left(G_{\text {read }}\right)$ was $0.011 \mathrm{~T} / \mathrm{m}$, and 200 complex points were collected per echo. Altogether 256 scans were accumulated in order to improve signal-to-noise ratio (SNR) due to low partial pressure of ${ }^{129}$ Xe. SNR was 19 in this experiment. Between the successive scans, the sample tube was refilled with a fresh hyperpolarized gas mixture. The repetition time was $10 \mathrm{~s}$ and the total experiment time $43 \mathrm{~min}$.

In the experiments of $\mathrm{Xe}$ adsorbed in $\mathrm{CPG}$, the following parameters were used: the duration and the bandwidth of the frequency-swept chirp pulse were $1 \mathrm{~ms}$ and $136254 \mathrm{~Hz}$. The diffusion delay $(\Delta)$ was $8.0 \mathrm{~ms}$ and the strength of the diffusion gradient ( $\left.G_{\text {diff }}\right)$ was $0.511 \mathrm{~T} / \mathrm{m}$. The echo time in the CPMG loop was $4 \mathrm{~ms}$, the strength of the read gradient $\left(G_{\text {read }}\right)$ was $0.026 \mathrm{~T} / \mathrm{m}$, and 100 complex points were collected per echo. Altogether 64 scans were accumulated in order to improve SNR ratio due to low partial pressure of ${ }^{129}$ Xe. SNR was 5 in this experiment. Between the successive scans, the 
sample tube was refilled with a fresh hyperpolarized gas mixture. The repetition time was $45 \mathrm{~s}$ and the total experiment time $48 \mathrm{~min}$.

The field-of-view was $16.5 \mathrm{~cm}$ in both experiments. It was intentionally set much larger than the size of the sensitive region of the coil (about $1.9 \mathrm{~cm}$ ), in order to get faster data sampling rate in the free induction decay (FID) signal due to digital filtering issues characteristic to Bruker instruments.

\section{Results and discussion}

2D contour plots of Fourier transformed data of the diffusion - $T_{2}\left(D-T_{2}\right)$ correlation experiments of hyperpolarized free Xe gas and Xe adsorbed in CPG materials are is shown in Figs. 2a and 2c. The Fourier transform was applied to extract MRI-type spatial encoding. The diffusion decay profile is shown in horizontal direction, in the relatively narrow region corresponding to the sensitive region of the coil. This region of the data was selected for 2D Laplace inversion. The $z$ axis was converted to the $b$ axis by using the linear relation between them (see ref. [16]).

$D-T_{2}$ correlation maps resulting from the $2 \mathrm{D}$ inverse Laplace transform are shown in Figs. $2 \mathrm{~b}$ and $2 \mathrm{~d}$. The maps include a single signal in both cases. The observed diffusion coefficient for the free gas is $(3 \pm 1) \cdot 10^{-6} \mathrm{~m}^{2} / \mathrm{s}$, which is close to the value reported for pure ${ }^{129} \mathrm{Xe}$ gas at $98 \mathrm{kPa},(5.3 \pm 0.6) \cdot 10^{-6} \mathrm{~m}^{2} / \mathrm{s}[34]$. The observed diffusion coefficient for xenon in CPG mesoporous material is $(2 \pm 1) \cdot 10^{-8} \mathrm{~m}^{2} / \mathrm{s}$, which is in a reasonable agreement with the value resulting from the standard pulsed-field-gradient stimulatedecho (PGSTE) experiment of the same HP xenon mixture in the same CPG sample, $(1.8 \pm 0.6) \cdot 10^{-8} \mathrm{~m}^{2} / \mathrm{s}$, and the values of xenon in similar Vycor glass, $(1.9-2.4) \cdot 10^{-8} \mathrm{~m}^{2} / \mathrm{s}$ 
[35]. The value is about two orders of magnitude smaller than that of free gas, due to the effect of adsorption. On the other hand, it is about an order of magnitude larger than the diffusion coefficient of Xe dissolved in water, $2 \cdot 10^{-9} \mathrm{~m}^{2} / \mathrm{s}[36]$.

The observed apparent $T_{2}$ values are very short, below $1 \mathrm{~ms}$, much shorter than $T_{2}$ values given by the standard, thermally polarized CPMG measurements, which were 2.6 and $6.3 \mathrm{~ms}$ for free and adsorbed gas (with Xe pressure of 5 bar), respectively. This is a consequence of combined effect of both the true $T_{2}$ relaxation and diffusion. In the ultrafast LNMR experiments, additional gradients are needed in the CPMG loop for reading the spatial encoding, and these gradients impose an additional diffusion weighting similar to standard spin echo diffusion experiment $[37,38]$. This effect is emphasized for gases with a large diffusion coefficient. In fact, because of this effect, in these experiments only the first CPMG echo produced a signal clearly above the noise level, and therefore the apparent $T_{2}$ value was not accurately observed, but only its upper limit. In principle, shortening the echo time in the CPMG loop could provide a remedy for this issue, but because the acquisition time of the echo signal has to be long enough in order to get high enough resolution for the magnetization profile image [3], it sets lower limits for the echo time.

The resolution in the $D-T_{2}$ maps in the $D$ direction is low, because of the low number of points in the diffusion encoding region in the magnetization profile images. In principle, the resolution could be improved either collecting echo signal for longer time or increasing read gradient strength [3]. However, both options would lead to additional loss of signal, which is not desired in these experiments. 


\section{Conclusions}

We demonstrated that ultrafast LNMR experiments, based on spatial encoding, are feasible with gas phase substances with standard solution-state NMR hardware. This broadens the application range of the LNMR method significantly; for example, many orders of magnitude larger diffusion coefficients of free and adsorbed gas are observed than those observed earlier in liquid state experiments $[16,23-25,27]$. On the other hand, fast diffusion of gas limited the resolution in the diffusion coefficient distribution in the $D-T_{2}$ experiment, and made the observed apparent $T_{2}$ very small. The resolution both in $D$ and $T_{2}$ direction could be slightly improved by keeping the read gradient on during the whole CPMG loop, also during the $\pi$ pulses. In this case, additional delays related to gradient ramping and stabilization could be removed. Several scans were accumulated in the experiments due to the low concentration of hyperpolarized ${ }^{129} \mathrm{Xe}$ in the gas mixture. If pure hyperpolarized Xe were collected in a cold finger and released into the sample, also single scan ultrafast LNMR experiments would become feasible. This would make ultrafast LNMR to an extremely useful method for the characterization of porous materials.

\section{Acknowledgements}

The financial support of Academy of Finland (grants \#289649 and 294027), University of Oulu Graduate School (UniOGS) and Emil Aaltonen Foundation is gratefully appreciated. The Laplace inversion program was provided by the late Prof. P. Callaghan. The CPG sample was kindly provided by the working group of Prof. D. Enke (University of Leipzig). 


\section{References}

1. J. Kowalewski, L. Maler, Nuclear Spin Relaxation in Liquids, CRP Press, Boka Raton, USA, 2006.

2. P.T. Callaghan, Translational Dynamics and Magnetic Resonance: Principles of Pulsed Gradient Spin Echo NMR, Oxford University Press, Oxford, USA, 2011.

3. B. Blümich, NMR Imaging of Materials, Oxford Univ. Press, Oxford, United Kingdom, 2000.

4. J. Kärger, R. Valiullin, Chem. Soc. Rev. 42 (2013) 4172-4197.

5. Y.-Q. Song, J. Magn. Reson. 229 (2013) 12-24.

6. P.M. Kekkonen, A. Ylisassi, V.-V. Telkki, J. Phys. Chem. C 118 (2014) 2146-2153.

7. D. Bernin, D. Topgaard, Curr. Opin. Colloind In. 18 (2013) 166-172.

8. L. Venkataramanan, Y.-Q. Song, M.D. Hürlimann, IEEE Trans. Signal. Proc. 50, (2002) 1017-1026.

9. Y.-Q. Song, L. Venkataramanan, M.D. Hürlimann, M. Flaum, P. Frulla, C. Straley, J. Magn. Reson. 154 (2002) 261-268.

10. J. Granwehr, P.J. Roberts, J. Chem. Theory Comput. 8 (2012) 3473-3482.

11. J.H. Ardenkjær-Larsen, B. Fridlund, A. Gram, G. Hansson, L. Hansson, M.H.

Lerche, R. Servin, M. Thaning, K. Golman, Proc. Natl. Acad. Sci. USA 100 (2003) 10158-10163.

12. S.R. Bowers, In: D.M. Gant, R.K. Harris (Eds.), Encyclopedia of Nuclear Magnetic Resonance, Wiley, Chichester, United Kingdom, 2002, p 750-769.

13. B.M. Goodson, J. Magn. Reson. 155 (2002) 157-216. 
14. D.A. Barskiy, A.M. Coffey, P. Nikolaou, D.M. Mikhaylov, B.M. Goodson, R.T. Branca, G.J. Lu, M.G. Shapiro, V.-V. Telkki, V. V. Zhivonitko, I.V. Koptyug, O.G. Salnikov, K.V. Kovtunov, V.I. Bukhtiyarov, M.S. Rosen, M.J. Barlow, S. Safavi, I.P. Hall, L. Schröder, E.Y. Chekmenev, Chem. Eur. J. 23 (2017) 725-751.

15. S. Ahola, V.-V. Telkki, ChemPhysChem 15 (2014) 1687-1692.

16. S. Ahola, V.V. Zhivonitko, O. Mankinen, G. Zhang, A.M. Kantola, H.-Y. Chen, C. Hilty, I. V. Koptyug, V.-V. Telkki, Nat. Commun. 6 (2015) 8363.

17. J.N. King, V.J. Lee, S. Ahola, V.-V. Telkki, T. Meldrum, Angew. Chem. Int. Ed. 55 (2016) 5040-5043.

18. L. Frydman, T. Scherf, A. Lupulescu Proc. Natl. Acad. Sci. USA 99 (2002) 1585815862.

19. P. Pelupessy J. Am. Chem. Soc. 125 (2003) 12345-12350.

20. A. Tal, L. Frydman, Prog. Nucl. Mag. Res. Sp. 57 (2010) 241-292.

21. L. Frydman, D. Blazina, Nature Phys. 3 (2007) 415-419.

22. N.M. Loening, M.J. Thrippleton, J. Keeler, R.G. Griffin, J. Magn. Reson. 164 (2003) 321-328.

23. M.J. Thrippleton, N.M. Loening, J. Keeler, Magn. Reson. Chem. 41 (2003) 441447.

24. S. Ahola, O. Mankinen, V.-V. Telkki, Magn. Reson. Chem. 55 (2016) 341-347. 25. Y. Shrot, L. Frydman, J. Magn. Reson. 195 (2008) 226-231.

26. P.E.S. Smith, K.J. Donovan, O. Szekely, M. Baias, L. Frydman, ChemPhysChem 14 (2013) 3138-3145.

27. L. Guduff, I. Kuprov, C. van Heijenoort, J.-N. Dumez, Chem. Commun., in press, DOI: $10.1039 / \mathrm{c} 6 \mathrm{cc} 09028 \mathrm{a}$. 
28. T.G. Walker, W. Happer, Rev. Mod. Phys. 69 (1997) 629-642.

29. E. Weilanda, M.-A. Springuel-Huet, A. Nossov, A. Gédéon, Micropor. Mesopor. Mat. 225 (2016) 41-65.

30. J. Demarquay, J. Fraissard, Chem. Phys. Lett. 136 (1987) 314-318.

31. V.V. Terskikh, I.L. Moudrakovski, S.R. Breeze, S. Lang, C.I. Ratcliffe, J.A.

Ripmeester, A. Sayari, Langmuir 18 (2002) 5653-5656.

32. J. Roukala, J. Zhu, C. Giri, K. Rissanen, P. Lantto, V.-V. Telkki, J. Am. Chem. Soc. 137 (2015) 2464-2467.

33. V.-V. Telkki, J. Lounila, J. Jokisaari, J. Chem. Phys. 124 (2006) 034711:1-8.

34. M. Pfeffer, O. Lutz, J. Magn. Reson. Ser. A, 113 (1995) 108-113.

35. I. L. Moudrakovski, A. Sanchez, C. I. Ratcliffe, J. A. Ripmeester, J. Phys. Chem. B $104(2000) 7306-7310$.

36. H. Weingärtner, R. Haselmeier, M. Holz, Chem. Phys. Lett. 195 (1992) 596-601.

37. P. Callaghan, J. Stepišnik, J. Magn. Reson. Ser A 117 (1995) 118-122.

38. J. Stepisnik, S. Lasic, A. Mohoric, I. Sersa, A. Sepe, J Magn Reson 182 (2006) 195 199. 


\section{FIGURE CAPTIONS}

Fig. 1. (a) Experimental setup, consisting of xenon hyperpolarizer and gas transport system into the NMR tube inside the NMR spectrometer. During the measurement, gas mixture flow was controlled by a valve system regulated via a solenoid valve (S). (b) Pulse sequence of ultrafast $D-T_{2}$ correlation experiment.

Fig. 2. Raw data of the $D-T_{2}$ correlation experiments, after the Fourier transform related to the image processing, for hyperpolarized free Xe (a) and Xe adsorbed in mesoporous CPG material (c), with the projections along the $T_{2}$ and $D$ directions. Corresponding $D-T_{2}$ correlation maps, resulting from the 2D Laplace inversion, are shown in (b) and (d). 


\section{FIGURES}

a)

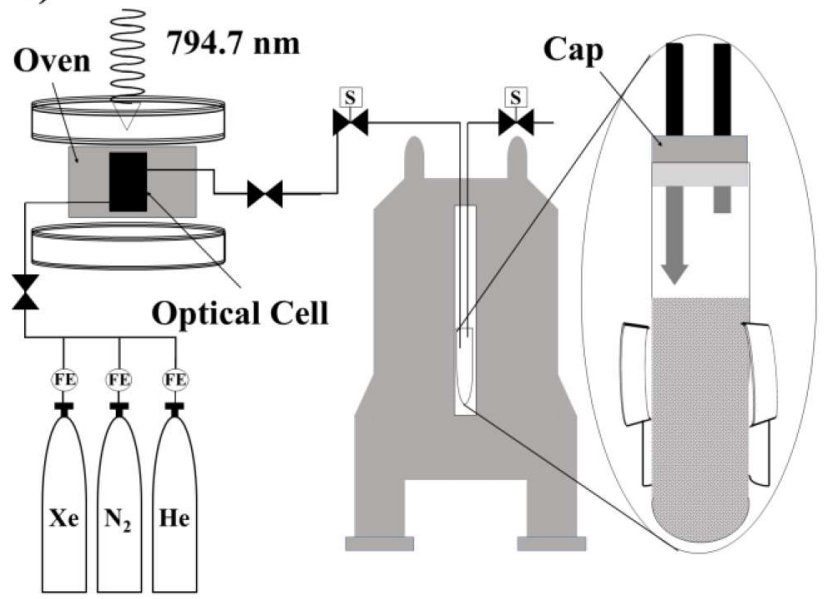

b)

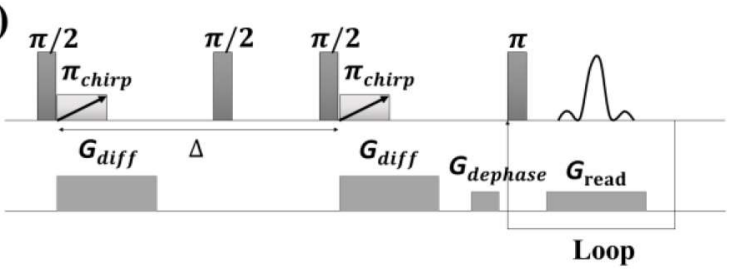

Fig. 1.

a)

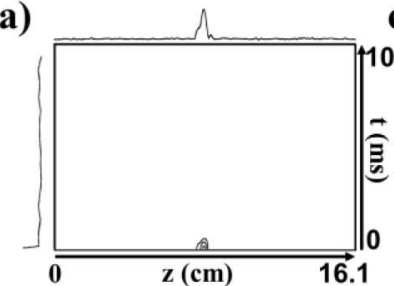

c)

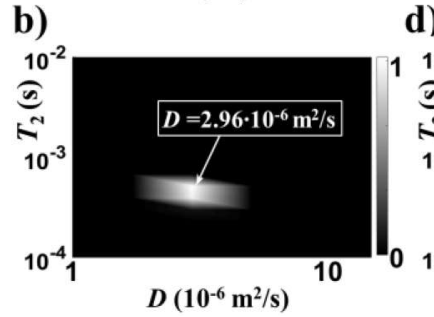

d)
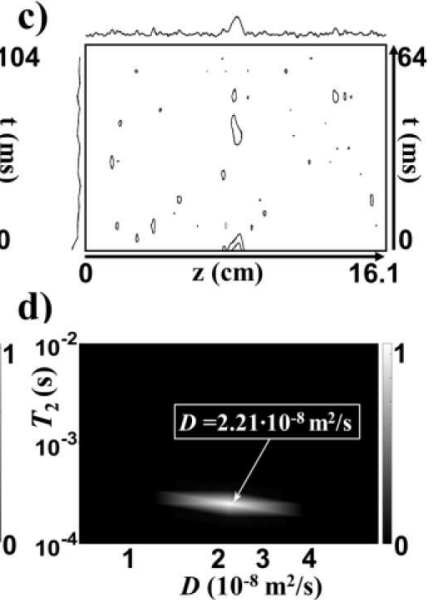

Fig. 2. 